\title{
Reducing uncertainties in a wind-tunnel experiment using Bayesian updating
}

\author{
D.J. Boon*, R.P. Dwight† J.J.H.M. Sterenborg, and H. Bijl \\ Aerodynamics Group, Delft University of Technology, The Netherlands
}

\begin{abstract}
We perform a fully stochastic analysis of an experiment in aerodynamics. Given estimated uncertainties on the principle input parameters of the experiment, including uncertainties on the shape of the model, we apply uncertainty propagation methods to a suitable CFD model of the experimental setup. Thereby we predict the stochastic response of the measurements due to the experimental uncertainties. To reduce the variance of these uncertainties a Bayesian updating technique is employed in which the uncertain parameters are treated as calibration parameters, with priors taken as the original uncertainty estimates. Imprecise measurements of aerodynamic forces are used as observational data. Motivation and a concrete application come from a wind-tunnel experiment whose parameters and model geometry have substantial uncertainty. In this case the uncertainty was a consequence of a poorly constructed model in the pre-measurement phase. These methodological uncertainties lead to substantial uncertainties in the measurement of forces. Imprecise geometry measurements from multiple sources are used to create an improved stochastic model of the geometry. Calibration against lift and moment data then gives us estimates of the remaining parameters. The effectiveness of the procedure is demonstrated by prediction of drag with uncertainty.
\end{abstract}

\section{Nomenclature}

$\begin{array}{ll}c & \text { Chord length }[\mathrm{m}] \\ c_{d} & \text { Drag coefficient }[-] \\ c_{l} & \text { Lift coefficient }[-] \\ c_{m} & \text { Moment coefficient }[-] \\ \boldsymbol{d} & \text { Experimental data vector } \\ e & \text { Observation noise } \\ h(\cdot) & \text { Regression functions } \\ t(\cdot) & \text { Covariance function } \\ m(\cdot) & \text { Cokriging mean function } \\ p(\cdot) & \text { Probability density } \\ \Delta q & \text { Geometric displacement of point } q[\mathrm{~m}] \\ x & \text { Position variable }[\mathrm{m}] \\ z & \text { Data vector } \\ B(\cdot) & \text { Bernstein polynomial } \\ \mathrm{CP} & \text { Free-Form Deformation control point } \\ D & \text { Aerodynamic drag force }[\mathrm{N}] \\ H & \text { Matrix of regression functions }\end{array}$

$\begin{array}{ll}L & \text { Aerodynamic lift force }[\mathrm{N}] \\ L(\cdot) & \text { Data likelihood function } \\ M & \text { Aerodynamic pitch moment }[\mathrm{Nm}] \\ \Delta P & \text { Control point displacement }[\mathrm{m}] \\ V & \text { Wind speed }[\mathrm{m} / \mathrm{s}] \\ V_{d} & \text { Data covariance matrix } \\ \alpha & \text { Angle of attack }\left[^{\circ}\right] \\ \Delta \alpha & \text { Angle of attack discrepancy }\left[^{\circ}\right] \\ \beta & \text { Regression parameters } \\ \delta & \text { Flap angle }\left[^{\circ}\right] \\ \Delta \delta & \text { Flap angle discrepancy }\left[^{\circ}\right] \\ \eta(\cdot) & \text { Computer model output } \\ \boldsymbol{\theta} & \text { Calibration parameters } \\ \sigma & \text { Standard deviation } \\ \epsilon & \text { Error } \\ \zeta(\cdot) & \text { Physical process }\end{array}$

\section{Introduction}

Aerodynamics experiments are sometimes performed for the purpose of providing validation data for CFD codes. Quantifying the uncertainty in the results of the experiment and simulation is important when

\footnotetext{
${ }^{*}$ Researcher, Faculty of Aerospace Engineering, P.O. Box 5058, 2600 GB Delft, The Netherlands.

${ }^{\dagger}$ Corresponding author: r.p.dwight@tudelft.nl
} 
performing any meaningful comparison of the two, and essential for CFD validation. The first aim of the current work is to investigate experimental input uncertainties and predict the uncertainties in the aerodynamic forces they lead to. The second aim is to use limited observations of the aerodynamics to reduce the variance in both parameters and forces. The first aim can be achieved by simulating the experimental setup with CFD, estimating parametric uncertainty from knowledge of the experiment, and finally propagating these uncertainties through this CFD model. The propagation can be performed with standard uncertainty quantification (UQ) techniques. The second aim can be achieved with Bayesian stochastic model updating, using the CFD model in the likelihood, and using Markov-chain Monte-Carlo (McMC) to sample the posterior. Once an improved model with reduced variability has been identified, it can be used to make predictions with uncertainty of unmeasured quantities.

A plethora of techniques exist for propagating an probability density function (pdf) on an input parameter through a computer code, to obtain pdfs on the outputs. Monte-Carlo involves taking random samples from the joint input parameter distribution and evaluating the CFD model at all these conditions. This approach has limited applicability to aerodynamic problems since the computational effort is generally large. Methods based on polynomial representations of the CFD response in the uncertain parameter space are much more efficient in low dimensional spaces, and applied here. In particular we use probabilistic collocation, 11 which has been applied to high-fidelity Navier-Stokes codes in Loeven et al.$^{[2]}$ and Chassaing and Lucor. ${ }^{3}$

Model calibration or model updating aims at improving the agreement between predictions and observations by identifying unknown physical and model parameters (calibration parameters). Stochastic model calibration does this in a Bayesian statistics setting, representing all uncertainties and errors as pdfs, and providing updated estimates of these pdfs given experimental observations. Bayesian calibration as this is known,, 4 requires defining prior pdfs on calibration parameters which represent our initial uncertainty in their values (prior to observations). The uncertainty propagation input distributions form a reasonable prior. We must then define the likelihood, the relationship between the CFD model predictions and the observed data. This term includes a stochastic term representing measurement noise, and perhaps others accounting for modelling inadequacy in the CFD, and is intended to capture all the reasons why we might not have perfect agreement between simulation and experiment. Given the prior and likelihood we can apply Bayes' theorem to obtain the posterior, representing our updated uncertainty on the calibration parameters given the data. The posterior may be evaluated with McMC methods.

The current research is performed within the context of a larger fluid-structure interaction (FSI) experimental and simulation programme. Prior to each FSI measurement, a steady measurement is performed to establish the steady characteristics of the experimental setup. Even the steady experiment is subject to uncertainties in the angle of attack, flap angle and wing geometry which result in large uncertainties in forces. As a consequence it is unreasonable to expect agreement between unsteady FSI experiments and predictions, unless the initial steady condition is reliably established.

The experimental and numerical modelling of the steady flow case is discussed in Section [1]. The investigation of uncertainties is based on uncertainty estimates of the input variables, e.g. the angle of attack and the wind speed. The input uncertainty estimates are obtained from direct measurements or expert judgement. A sensitivity analysis is performed to determine the uncertain parameters most important to the observations. The probability distributions of the important uncertain parameters are propagated through a computer simulation model to obtain uncertainty characteristics of the aerodynamic quantities, see Section III] Using the experimental observations the computer model is calibrated to improve on the agreement of predictions with experimental observations and to reduce the simulation output uncertainties, as discussed in Section IV

\section{Aerodynamic flow case}

The flow case under consideration consists of steady flow about a wing equipped with a trailing edge flap.

The flow case is investigated by performing an experiment in a wind tunnel. Computations are performed using a numerical simulation code, against whose predictions the experimental observations are compared. The uncertainty that are present in the experiment is investigated using the simulation code. 


\section{II.A. Experiment}

The aerodynamic experiment is performed in a low speed low turbulence wind tunnel. The wind tunnel has a closed test section with a contraction ratio of 17.8 to 1, yielding a low free stream turbulence level of about $0.015 \%$ at a wind speed of $21 \mathrm{~m} / \mathrm{s}$. The $2.6 \mathrm{~m}$ long octagonal test section has a width of $1.8 \mathrm{~m}$ and a height of $1.25 \mathrm{~m}$. The tunnel is equipped with a six-component balance system to measure forces and moments.

The experiment is performed at a wind speed of $21 \mathrm{~m} / \mathrm{s}$ and a Reynolds number of 700000 . The wind tunnel model consists of an untwisted and untapered wing with a $0.2 \mathrm{c}$ deflectable trailing edge flap. The wing is manufactured according to the $18 \%$ thick DU96-W-180 wind turbine airfoil, with a manufacturing tolerance of about $1 \mathrm{~mm}$. The wing has a chord of $0.5 \mathrm{~m}$ and a span of $1.8 \mathrm{~m}$. During the steady measurements the flap angle is fixed at zero degrees. The wing is horizontally attached to an external frame with an electronic rotation system to adjust the angle of attack. The angle of attack ranges from -6 to 6 degrees such that the flow stays attached to the wing surface and flow blockage effects are small.

Corrections are applied to the measured aerodynamic forces and moments to correct for the presence of wind tunnel walls ${ }^{6}$ Furthermore, following Timmer ${ }^{7}$ a correction is applied to cope with three-dimensional effects due to the presence of small gaps between the walls and the wing on both sides. The corrected experimental results are compared with two-dimensional numerical simulations without tunnel walls.

\section{II.B. Numerical simulations}

The experiment is numerically investigated using the XFOIL simulation code $\frac{8}{8}$ XFOIL is an enhanced panel method based flow solver for two-dimensional subsonic steady flows. It employs an inviscid formulation to solve the outer domain and boundary layer equations to solve the viscous inner domain. The inviscid formulation of XFOIL is a linear-vorticity stream function panel method to solve steady incompressible flow problems. Compressibility is included by applying a compressibility correction for Mach numbers up to sonic conditions. Viscosity is taken into account in the region near the airfoil surface and in the wake by employing an integral boundary layer formulation. Boundary layer transition is predicted employing the $e^{N}$ method $\underline{9}^{9}$

XFOIL is particularly useful for uncertainty analysis since it has a short evaluation time of less than one to a few seconds. A two-dimensional simulation code is suitable for simulating the steady flow case, since our experiment is corrected for three-dimensional effects and comprises attached flow. A summary of the XFOIL input settings is presented in Table 1. The input airfoil consists of a deformation of the DU-96-W180 profile using the mean of the FFD distribution. The FFD distribution is a representation of the measured three-dimensional wing geometry, as described in section III.

\begin{tabular}{lr}
\hline \hline Geometry & \\
Airfoil & $\begin{array}{r}\text { DU96-W-180 } \\
\text { (measured) }\end{array}$ \\
Number of panels & 350 \\
\hline Flow conditions & \\
Mode & viscous \\
Reynolds number Re & $7 \cdot 10^{5}$ \\
Mach number Ma & 0.061 \\
\hline Boundary layer & \\
Amplitude ratio & 11 \\
\hline \hline
\end{tabular}

Table 1: XFOIL input settings for the discretized geometry, flow conditions and boundary layer parameters.

\section{Uncertainty quantification}

A stochastic investigation is performed on the aerodynamic experiment, which is subject to uncertainties in the input variables. Various uncertain input variables can be identified in the experiment such as the wing geometry, the angle of attack and the wind speed. The choice of uncertain variables to be stochastically 
modeled is made based on their expected importance to the results. The uncertain geometry and the uncertain experimental variables are discussed in separate sections. Estimates of the input uncertainties are made based on measurements of the uncertain variables and on measuring device tolerances. Once the uncertainty distributions are obtained for the uncertain variables a sensitivity analysis is performed to reduce the amount of uncertain variables to be taken into account for the uncertainty analysis. The reduction in amount of uncertain variables consists of neglecting the less important variables in order to reduce the computational effort. The uncertainty distributions of the most important variables are propagated through a computer simulation code to provide the stochastic response of the aerodynamic outputs. Finally, the experimental observations are compared with the numerical simulations.

\section{III.A. Uncertain wing geometry}

The wing model has been manufactured according to the DU96-W-180 airfoil with a manufacturing tolerance of about $1 \mathrm{~mm}$. Because of the fact that geometric variations in the order of $1 \mathrm{~mm}$ can induce significant variations in the aerodynamic forces, the wing geometry is regarded as an uncertain input. Based on geometric measurements the uncertainty distribution of the wing geometry is determined. In order to be able to perform an uncertainty analysis the wing geometry is parameterized and the uncertainty distribution for the parameterization is computed.

\section{III.A.1. Geometry measurements}

In order to investigate the influence of geometric uncertainties on the aerodynamic forces the wing geometry has been measured using two different techniques. A coordinate measuring machine (CMM) is employed to measure the wing geometry surface along a straight line at seven locations along the wingspan. The machine scans the wing surface with a ball roller attached to a moving measurement arm. The resulting two-dimensional profiles are subject to a measurement uncertainty of about $0.25 \mathrm{~mm}$.

A separate optical technique called photogrammetry is used to obtain three-dimensional coordinates of the wing object. This technique is based on combining information from several pictures taken with calibrated cameras at various angles with respect to the object. The object is marked with stickers to identify specific locations. A computer algorithm extracts the information from the pictures and yields coordinates of the three-dimensional object. The accuracy of the photogrammetry measurements amounts to $0.10 \mathrm{~mm}$.

\section{III.A.2. Wing model}

The CMM measurements are combined with the three-dimensional wing object coordinates obtained by photogrammetry into one geometric model using cokriging. Kennedy and O'Hagan $\frac{10}{10}$ explain cokriging as a technique employing Gaussian processes for the interpolation of outputs by combining various data sources, taking into account the uncertainty of each input data point and the smoothness of the data. Practically the cokriging interpolated value $m(x)$ at location $x$ is obtained from

$$
m(x)=h(x)^{T} \beta+t(x)^{T} V_{d}^{-1}(z-H \beta),
$$

where $h(x)^{T}$ is a vector of regression functions and $\beta$ a vector of corresponding regression parameters. The matrix $H$ is obtained from evaluating $h(x)^{T}$ for all locations corresponding to the input data in data vector $z$. A weight matrix is formed by the product of the covariance between data and interpolation location $t(x)^{T}$ and the data covariance matrix $V_{d}$. Following the work of Higdon et a ${ }^{111}$ the observation error is included in the data covariance matrix $V_{d}$. Estimates for the interpolation uncertainty can be obtained from the interpolation covariance function. 10

Cokriging enables to combine the relevant information from the low accuracy CMM measurements with the high accuracy photogrammetry measurements. The CMM measurements provide high resolution data of geometric variations in the chordwise direction, although the overall accuracy is low. The photogrammetry measurements provide accurate information about three-dimensional coordinates of the wing, although the resolution is low. Including both data sources into one model by employing cokriging enables to combine high resolution geometric variations from the CMM measurements with the accuracy of the photogrammetry measurements. Figure 1 shows the measurements of the geometry and the wing model obtained using cokriging. 


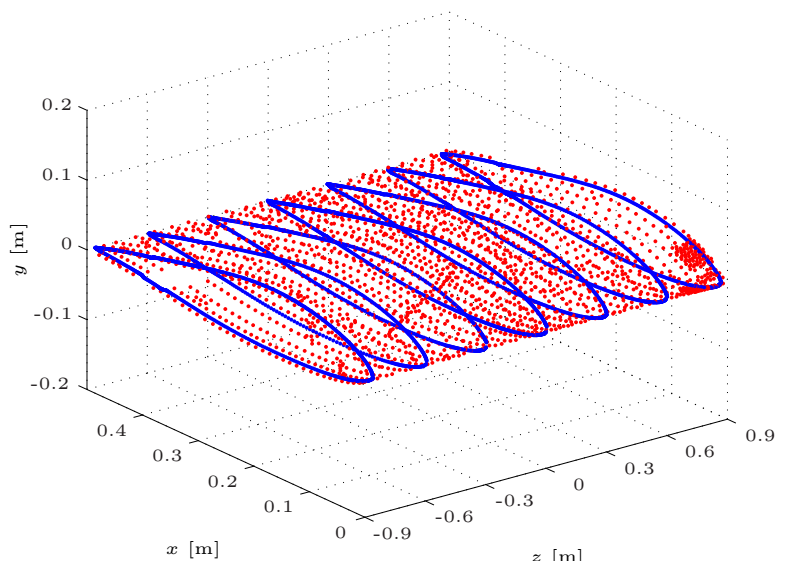

(a) Geometry measurements.

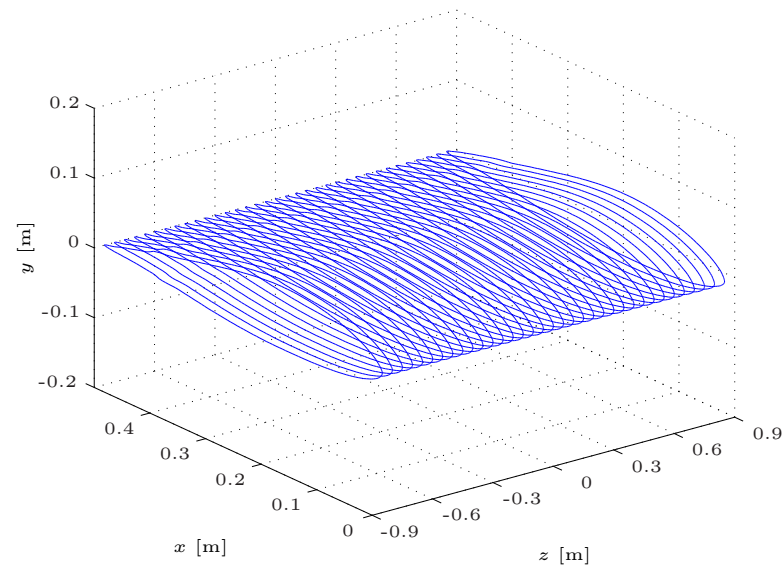

(b) Cokriging wing model.

Figure 1: CMM measurements (blue dots) and photogrammetry measurements (red dots) of the threedimensional wing geometry are shown in subfigure (a). The three-dimensional wing model resulting from cokriging of the CMM and photogrammetry data is shown in subfigure (b).

\section{III.A.3. Free-form deformation}

The wing model obtained using cokriging is subject to measurement and interpolation uncertainties. In order to be able to investigate the influence of these uncertainties on the aerodynamic forces the three-dimensional wing object needs to be parameterized. A free-form deformation (FFD) technique is employed according to Duvigneau ${ }^{12}$ because of its ability to effectively parameterize deviations of the wing geometry from the DU96-W-180 airfoil shape.

The FFD technique allows the deformation of an object in two-dimensional or three-dimensional space regardless of its geometrical description. A quadrilateral lattice is built around the object to be deformed. The FFD action can be represented by the movement of control points, which are located on the sides of the lattice. By moving the control points, the object inside the lattice is deformed. A local coordinate system $(\xi, \eta)$ is defined in the lattice with $(\xi, \eta) \in[0,1] \times[0,1]$. The deformation step consists of computing the displacement $\Delta q$ of each point $q$ inside the lattice as defined by the second-order Bézier tensor product

$$
\Delta q=\sum_{i=0}^{n_{i}} \sum_{j=0}^{n_{j}} B_{i}^{n_{i}}\left(\xi_{q}\right) B_{j}^{n_{j}}\left(\eta_{q}\right) \Delta P_{i j},
$$

where $B_{i}^{n_{i}}$ and $B_{j}^{n_{j}}$ are the Bernstein polynomials of order $n_{i, j}$ defined by

$$
B_{p}^{n}(t)=C_{n}^{p} t^{p}(1-t)^{n-p} .
$$

The order $n_{i, j}$ of the Bernstein polynomials is equivalent to the amount of control points on the sides of the lattice. The $\left(\xi_{q}, \eta_{q}\right)$ coordinates in Equation (11) are the FFD coordinates of the point $q$, which result from describing the object in lattice coordinates. The weighting coefficients $\Delta P_{i j}$, i.e. the control points displacements, are the driving variables that induce the deformation.

In order to keep the amount of parameters low the choice is made to represent the three-dimensional wing model using the FFD in two dimensions. A compromise between the ability to represent the airfoil and the computational effort of the uncertainty analysis result in an amount of FFD parameters of 10 control points $\mathrm{CP}_{1,2, \ldots, 10}$.

\section{III.A.4. Parameter uncertainty distribution}

For the uncertainty analysis a distribution needs to be determined on the FFD parameters that represents the uncertain wing geometry. Since the FFD is a linear operator the FFD control points corresponding to a certain airfoil can easily be found by applying a least squares approximation. Since this approach is computationally cheap a Monte Carlo approach suffices to compute the FFD parameter distribution. 
First, a large number of two-dimensional profiles is generated from the cokriging wing model. The large number is determined to be $10^{5}$ samples by checking the convergence of the Monte Carlo approach. These profiles are evenly distributed along the wing span and can be represented by a two-dimensional airfoil distribution. Applying the least squares approach to all the two-dimensional profiles results in $10^{5}$ FFD parameter samples. Assuming the FFD parameter distribution to be multivariate normal the characteristics are obtained by taking the sample mean and covariance of the $10^{5} \mathrm{FFD}$ parameter samples.

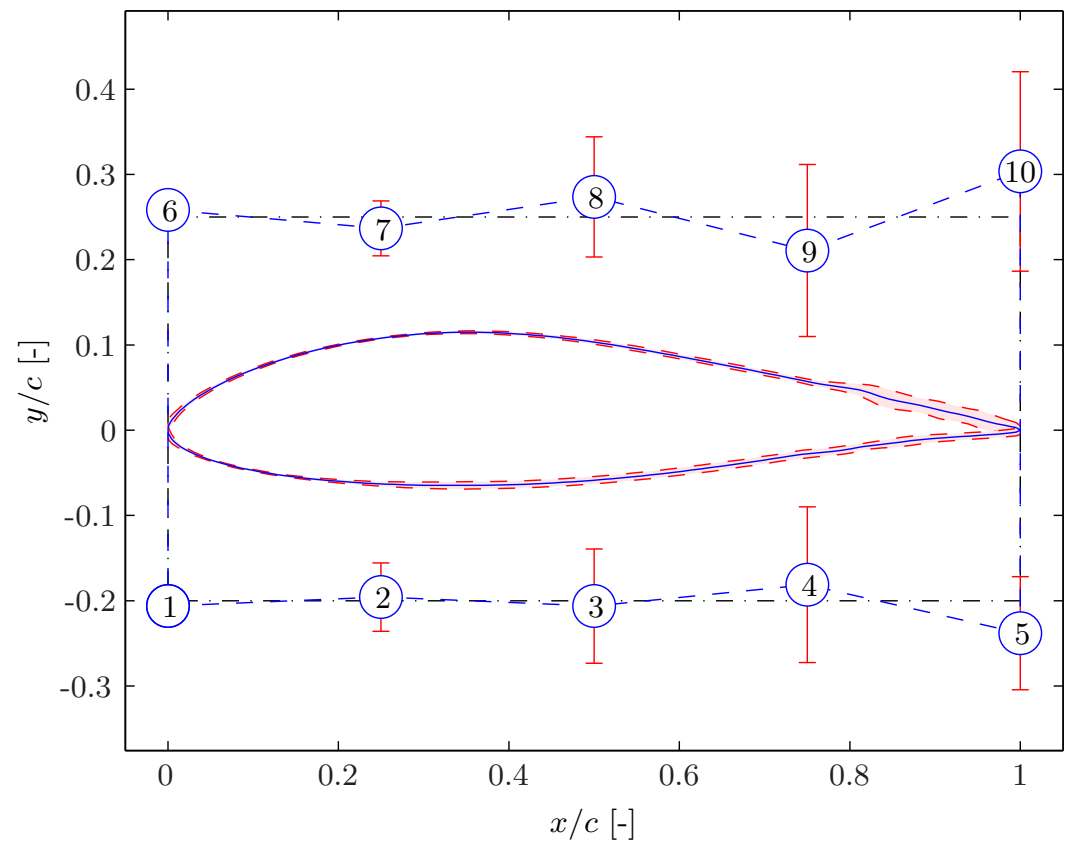

Figure 2: Two-dimensional airfoil distribution and FFD parameter distribution. The mean of the uncertainty distributions is shown in blue and the standard deviation in red. The shaded area around the airfoil indicates an uncertainty interval of 5 standard deviations and the error bars on the control points indicate uncertainty intervals of 2 standard deviations. The black dashdotted line indicates the zero control point deflection, which corresponds to the original DU96-W-180 geometry.

The resulting FFD parameter distribution and the two-dimensional airfoil distribution are shown in Figure 2. The geometric uncertainties as well as the interpolation uncertainties have been transformed into uncertainties in the FFD control points. The uncertainty is largest in the flap region of the airfoil for both the two-dimensional airfoil distribution as well as the FFD control point distribution.

\section{III.B. Uncertain experimental variables}

In the experimental setup many variables are present that are uncertain in some sense. The important uncertain variables need to be selected and the less important ones neglected for the uncertainty analysis to reduce computational effort. Variables such as the static pressure, density and kinematic viscosity are accurately measured and have a relatively low influence on the aerodynamic forces. The angle of attack, flap angle, chord length and wind speed however are believed to be important uncertain variables and are therefore selected for the sensitivity analysis.

Assuming normal distributions the uncertainties are derived for the uncertain variables by reviewing the procedure of measuring the variables. During the experiment the angle of attack $\alpha$ and flap angle $\delta$ are set by making use of a mould that is made based on geometric measurements of the wing at midspan. Due to possible movement of the mould with respect to the wing uncertainties arise in the angles. The total uncertainty in angle of attack consists of a sum of mould inaccuracy and measurement device errors and amounts to 0.25 degrees. The uncertainty in the measured flap angle is mainly caused by the inaccurate mould, but has also contributions from dynamic movement in the attachment system and measurement device errors. The total uncertainty in flap angle amounts to 0.59 degrees. An estimate of the uncertainty in the chord length $c$ is obtained from examining the geometric measurements. The standard deviation is determined as $2.5 \mathrm{~mm}$ based on variations of the chord length along the wing span. Point measurements of 
the wind speed $V$ are available at each entry in the angle of attack polar. The sample standard deviation of these wind speed data is computed as $0.05 \mathrm{~m} / \mathrm{s}$ and is used to determine the uncertainty distribution of the wind speed.

\section{III.C. Sensitivity analysis}

A sensitivity analysis is performed to further reduce the amount of uncertain variables taken into account in the uncertainty analysis by identifying the important parameters. The uncertainty distributions of the experimental variables and the FFD parameters are the input for the sensitivity analysis. The sensitivity analysis is based on the scaled sensitivity derivative according to the method presented by Loeven and Bijl! 13 The sensitivity derivative is determined using the probabilistic collocation (PC) method and is defined as the partial derivative of any output of interest $u$ with respect to an uncertain parameter $a$. The sensitivity derivative multiplied with the standard deviation to obtain the scaled sensitivity derivative as $\sigma_{a} \frac{\partial u}{\partial a}$. The derivatives are computed by differentiating a second order PC approximation of the outputs of interest with respect to each parameter separately. The PC approximation is based on evaluations of the XFOIL simulation code.

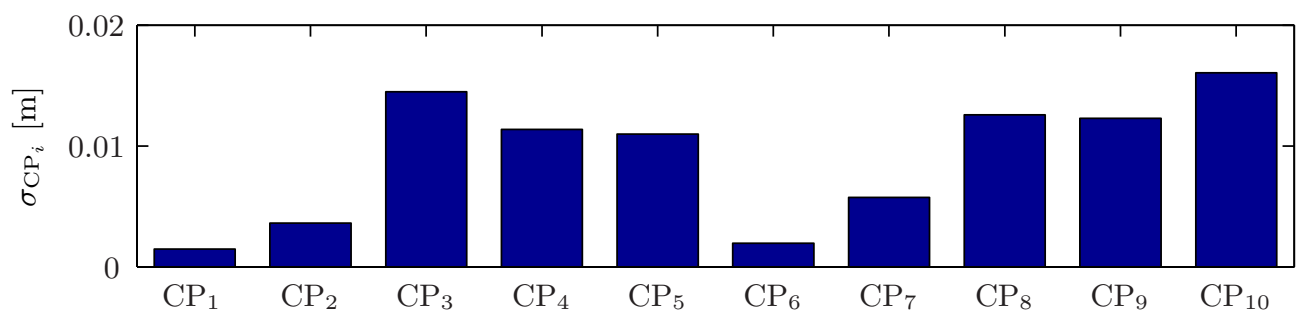

(a) Standard deviation of the uncertainty distributions

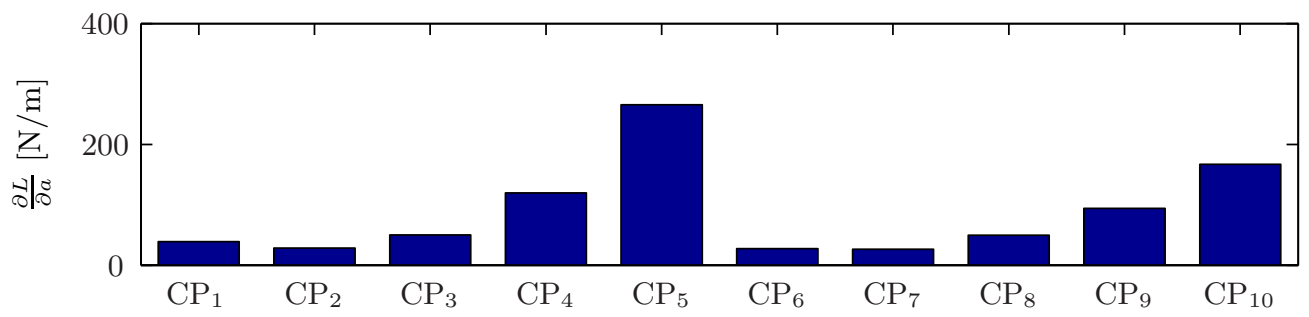

(b) Sensitivity derivative of the lift

Figure 3: Standard deviations of the uncertainty distributions of the control points and sensitivity derivatives of the lift $L$ with respect to the control points. For the specific locations of the numbered control points see Figure 2,

The sensitivity of the lift coefficient output with respect to the parameterized geometry is shown in Figure 3. In this figure the standard deviation of the uncertainty distributions of the control points is shown together with the unscaled sensitivity derivative. As stated earlier the uncertainty in the wing geometry is largest in the flap region of the airfoil. Furthermore, the lift force is most sensitivity to geometric variations in the flap region as well. Because the uncertainty as well as the sensitivity are both highest in the flap region it can be concluded that the flap region is most influential for the stochastic response of the outputs.

A good estimate of the effect of the uncertain parameter on the output of interest is obtained by scaling the sensitivity derivatives with the standard deviation of the distributions of the uncertain parameters. In case of $N$ uncertain parameters, the most important parameter is determined by

$$
\max \left\{\sigma_{a_{1}} \frac{\partial u}{\partial a_{1}}, \sigma_{a_{2}} \frac{\partial u}{\partial a_{2}}, \ldots, \sigma_{a_{N}} \frac{\partial u}{\partial a_{N}}\right\} .
$$

The scaled sensitivity derivatives for the aerodynamic lift and moment are shown in Figure 4 The uncertain parameters that have a negligible influence on both the lift and moment are the wind speed $V$, chord length $c$ and the four FFD control points $\mathrm{CP}_{1,2,6,7}$. Their influence on the total stochastic response of the lift and moment is less than $1 \%$. The remaining parameters are regarded as important for the stochastic 


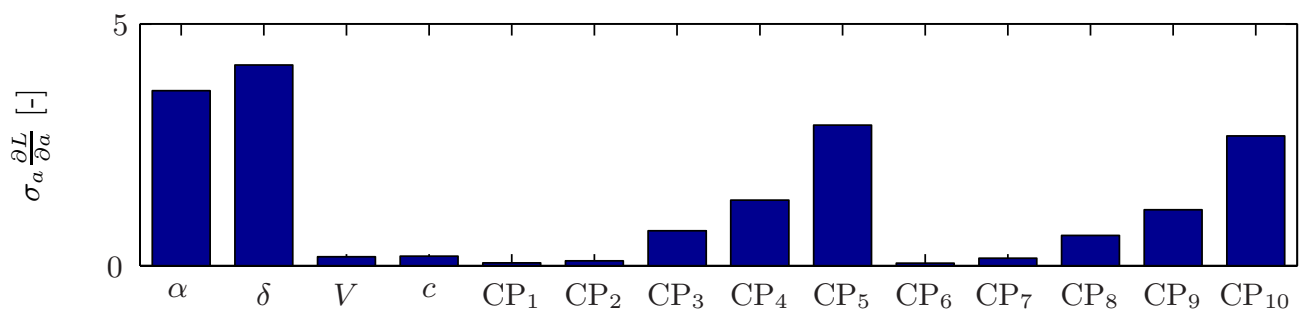

(a) Scaled sensitivity derivative of the lift

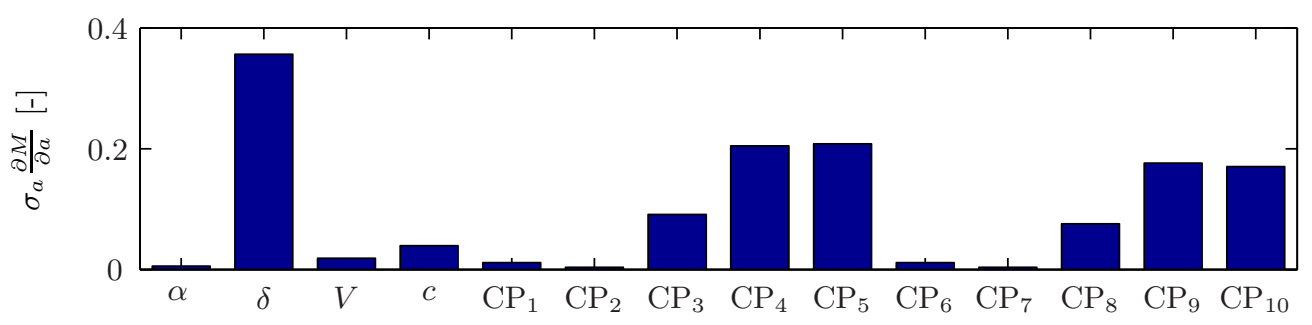

(b) Scaled sensitivity derivative of the moment

Figure 4: Scaled sensitivity derivatives of the lift $L$ (a) and moment $M$ (b) with respect to the uncertain input parameters $a$.

response of the aerodynamic forces and moments and will therefore be used as inputs for the uncertainty propagation.

\section{III.D. Uncertainty analysis}

The sensitivity analysis has shown that the most important variables are the angle of attack $\alpha$, flap angle $\delta$ and the FFD control points $\mathrm{CP}_{3,4,5,8,9,10}$. The probability distribution functions of these variables are propagated using the probabilistic collocation method to obtain the stochastic response of the lift and moment coefficients. A PC approximation is built using deterministic computations of the XFOIL simulation code.

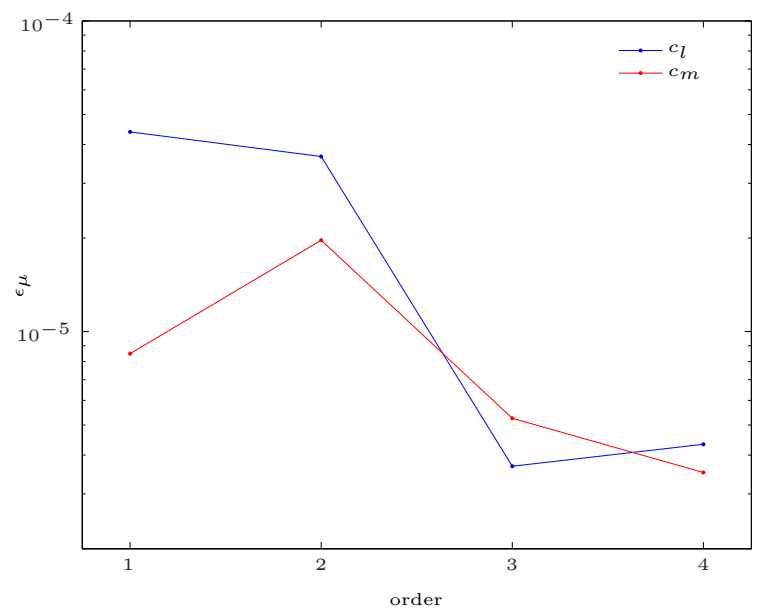

(a) Mean.

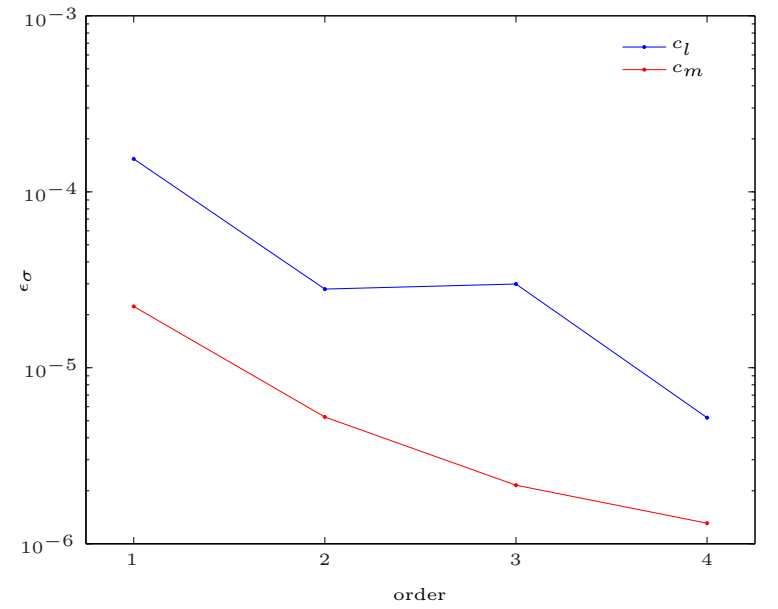

(b) Standard deviation.

Figure 5: Approximation error estimates for the mean and standard deviation of various orders of probabilistic collocation approximations.

A convergence study is performed to determine the accuracy of the PC approximation. The accuracy is checked by comparing PC approximations of order 1 to 4 with a PC approximation of order 5 . Based on the assumption that the convergence is similar for the various FFD parameters, only a subset of the control 
points is used for the convergence study to reduce computational effort. Thus, PC approximations are built using the variables angle of attack, flap angle and the control points $\mathrm{CP}_{8,10}$. The approximation errors for the mean $\epsilon_{\mu}$ and the standard deviation $\epsilon_{\sigma}$ are computed as

$$
\begin{aligned}
\epsilon_{\mu} & =\left|\mu^{P}-\mu^{P_{r e f}}\right| \\
\epsilon_{\sigma} & =\left|\sigma^{P}-\sigma^{P_{r e f}}\right|,
\end{aligned}
$$

where $P$ is the $\mathrm{PC}$ approximation order and $P_{\text {ref }}$ denotes the order $5 \mathrm{PC}$ approximation, which is used as the reference. The PC approximation is considered to be sufficiently accurate when the approximation error is much smaller than the expected standard deviation of the stochastic response of the outputs. A rough estimate of the expected output standard deviation for the lift coefficient due to uncertain angle of attack can be obtained using thin airfoil theory as $2 \pi \sigma_{\alpha} \approx 0.03$. Figure 5 shows that a PC approximation of order 1 is sufficiently accurate since the approximation errors for the mean and for the standard deviation are both more than 100 times smaller than the expected output standard deviation. Furthermore, the graph shows that for a PC approximation of order 3 the approximation errors for the mean and standard deviation are both smaller than the XFOIL rounding error of $0.5 \cdot 10^{-4}$ for the lift coefficient and $0.5 \cdot 10^{-5}$ for the moment coefficient.

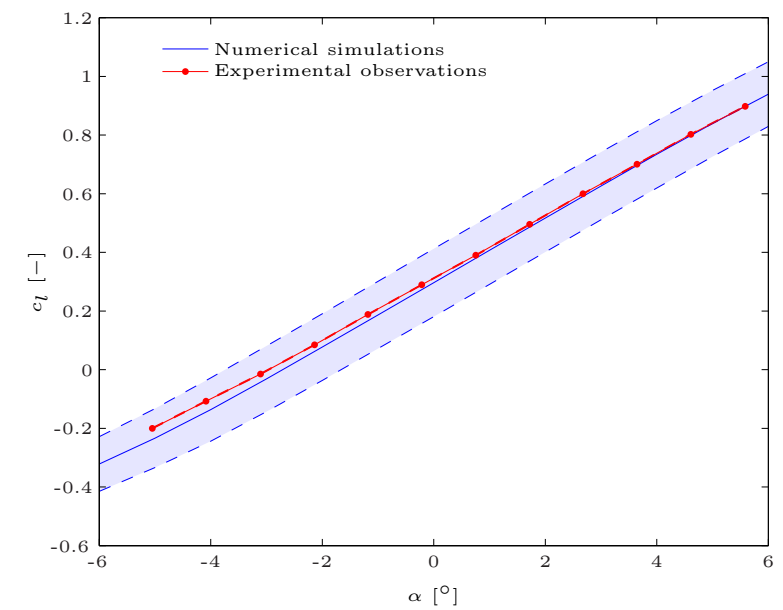

(a) Lift coefficient.

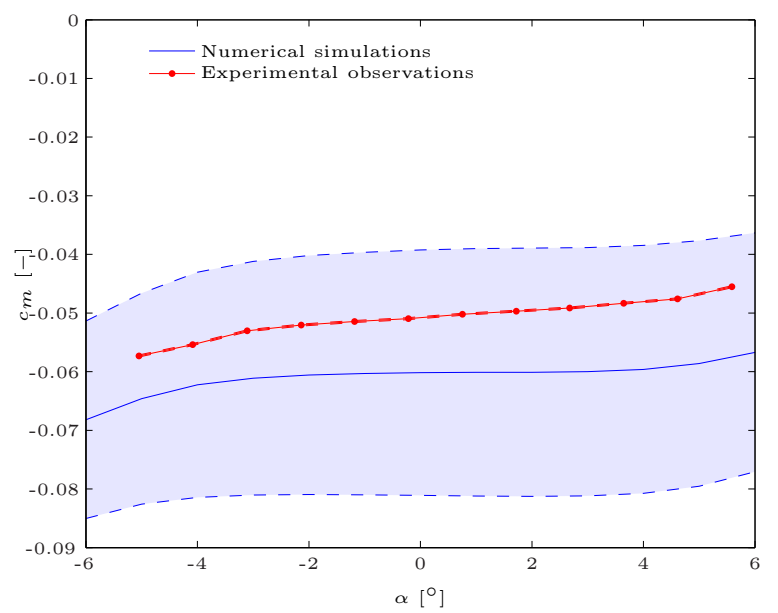

(b) Moment coefficient.

Figure 6: Comparison of the uncertain lift coefficient and moment coefficient with experimental observations. The solid lines indicate the deterministic values and the shaded areas indicate an uncertainty interval of 1 standard deviation.

The stochastic response of the outputs due to the uncertain inputs is obtained using a first order PC approximation. For a first order approximation with 8 uncertain input variables $(1+1)^{8}=256$ deterministic evaluations are required at each point in the $c_{l}-\alpha$ curve. The mean and variance of the outputs of interest can be directly obtained from the deterministic outcomes and the PC weights. $\frac{13}{13}$ Figure 6 shows the results of the uncertainty analysis for the lift coefficient and moment coefficient as a function of angle of attack. The measurements are shown in red with the measurement error indicated as an uncertainty interval. The measurement error is determined by the uncertainty of the measurement device, i.e. the six-component balance system. The average uncertainty standard deviation amounts to 0.11 and 0.02 for the lift and moment coefficient respectively. The fact that the experimental observations lie within the uncertainty range of the simulations indicates the possibility that the discrepancy between observations and simulations is caused by input uncertainties. However, it is likely that simulation model inadequacy also plays a role in causing the discrepancy.

\section{Data assimilation}

Data assimilation is employed to reduce the uncertainty levels in the numerical simulations and improve on the agreement between simulations and experimental observations. Bayesian calibration enables to make 
inferences on the observations about the numerical simulations. Calibration employs Bayesian updating to make estimates of uncertain code input variables based on experimental observations. Observational data of the lift coefficient and moment coefficient are used to calibrate the simulation model. Simulations of the calibrated model show improved agreement with the observations and considerably smaller uncertainty levels. Predictions of the drag coefficient that are obtained using the calibrated simulation model show good agreement with the observational data.

\section{IV.A. Bayesian calibration}

The uncertainty analysis has provided output uncertainty characteristics by propagating input uncertainty estimates through a simulation model, i.e. the XFOIL code. Treating the problem in a reverse manner by making inferences about the simulation model using the observations is known as the inverse problem. Kennedy and O'Hagan ${ }^{4}$ came up with a framework for Bayesian calibration that offers unique capabilities to obtain information about the objects of inference, i.e. the uncertain inputs, by making use of observations. The goal of calibration is to improve on the simulation code predictions.

Calibration refers to estimating unknown or uncertain code input parameters such that the observational data $z_{i}$ fit the simulation code outputs as closely as possible. The relationship between the observations $z_{i}$, the true physical process $\zeta$ and the computer model output $\eta$ is represented by

$$
z_{i}=\zeta\left(\boldsymbol{x}_{i}\right)+e_{i}=\eta\left(\boldsymbol{x}_{i}, \boldsymbol{\theta}\right)+\epsilon_{i},
$$

where $e_{i}$ is the observation error for the $i^{\text {th }}$ observation. The observation error is assumed to be normally distributed with zero mean and standard deviation $\lambda$, i.e. $e_{i} \sim N(0, \lambda)$. The simulation residual variation is denoted by $\epsilon_{i}$. A distinction is made between the variable inputs $\boldsymbol{x}_{i}$ and the calibration parameters $\boldsymbol{\theta}$, i.e. the input parameters to be calibrated. The true calibration parameters $\boldsymbol{\theta}$ provide the best fit of the simulation data to the observations according to the error structure of the statistical model. Usually an extra term $\kappa\left(\boldsymbol{x}_{i}\right)$ is included in the right hand side of equation 2 to account for model inadequacy. However, since only little uncorrelated observation data is available it is impossible to obtain a Gaussian process model of the discrepancy term. Therefore the discrepancy term is not included in the current work.

The calibration estimates for $\boldsymbol{\theta}$ are obtained from exploring the posterior distribution. The full joint posterior distribution is obtained from applying Bayes' theorem to the statistical model as

$$
p\left(\boldsymbol{\theta} \mid \boldsymbol{d}_{C_{L}}, \boldsymbol{d}_{C_{M}}\right) \propto L\left(\boldsymbol{d}_{C_{L}} \mid \boldsymbol{\theta}\right) L\left(\boldsymbol{d}_{C_{M}} \mid \boldsymbol{\theta}\right) p(\boldsymbol{\theta}),
$$

where $L\left(\boldsymbol{d}_{C_{L}} \mid \boldsymbol{\theta}\right)$ and $L\left(\boldsymbol{d}_{C_{M}} \mid \boldsymbol{\theta}\right)$ are the data likelihood functions corresponding to the lift coefficient and moment coefficient data. The full data vectors $\boldsymbol{d}_{C_{L, M}}$ are modeled as Gaussian processes conditional on the calibration parameters $\boldsymbol{\theta}{ }^{11}$ The prior distributions $p(\boldsymbol{\theta})$ are assumed to be normally distributed.

The available experimental observations are the lift coefficient, drag coefficient and moment coefficient. In order to be able to show the succes of the calibration the drag coefficient data is not used for calibration but afterwards for comparison with calibrated predictions. The experimental observations for calibration are therefore limited to the $c_{l}-\alpha$ curve and the $c_{m}-\alpha$ curve. Since the lift and moment coefficient curves can be approximated by linear functions of $\alpha$ it means that only 3 or 4 pieces of independent data are available and thus 3 or 4 unknown variables can be estimated by calibration. However, the amount of important uncertain variables is larger than the amount of independent data in the experimental observations. Therefore, the amount of uncertain variables to be calibrated for is reduced by assuming that the geometric variations can be modeled by angle of attack and flap angle discrepancies. Based on this assumption the total input uncertainty can be incorporated in an angle of attack discrepancy $\Delta \alpha$ and a flap angle discrepancy $\Delta \delta$. Calibration is performed to obtain estimates for these uncertain variables, i.e. the calibration parameters. Rough estimates are made of the prior distributions for the calibration parameters with mean zero and a standard deviation of 1 degree for both the angle of attack and flap angle discrepancy. The XFOIL simulation code is employed to provide the computer model outputs $\eta$. The simulation code outputs $\eta()$ are modeled using Gaussian processes.

Markov chain Monte Carlo (McMC) simulation is used to explore the joint posterior distribution and obtain estimates for the calibration parameters. The Markov chain shows convergence for a total chain size of $10^{5}$ samples. The part of the Markov chain that does not belong to the stationary distribution, which is also known as the burn in, is determined as the initial $10^{4}$ samples. Taking the sample mean and covariance of the stationary distribution in the Markov chain yields the calibration estimates. The calibration estimates 
are presented in Table 2 and the covariance between the calibration parameters is visualized in Figure 7 The calibration has reduced the prior estimates of the uncertainty expressed in standard deviations of 1 degree to standard deviations of 0.04 and 0.07 degree for the angle of attack discrepancy and flap angle discrepancy respectively. These uncertainty results are also considerably smaller than the estimates for the uncertain input parameters as discussed in section III. It can be concluded that the uncertainty estimates for the important uncertain input variables have been reduced to small uncertainties represented by an angle of attack discrepancy and flap angle discrepancy.

\begin{tabular}{lcc}
\hline \hline & mean & standard deviation \\
\hline angle of attack discrepancy $\Delta \alpha$ & $0.672^{\circ}$ & $0.038^{\circ}$ \\
flap angle discrepancy $\Delta \delta$ & $-1.009^{\circ}$ & $0.067^{\circ}$ \\
\hline \hline
\end{tabular}

Table 2: Calibration results for the uncertain parameters.

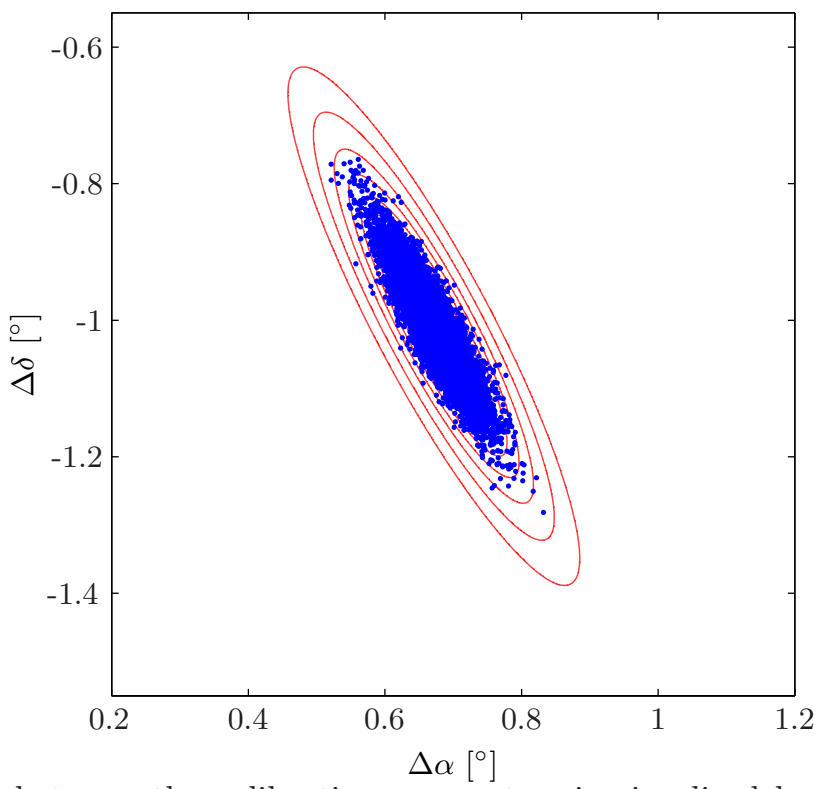

Figure 7: The covariance between the calibration parameters is visualized by plotting the Markov chain samples in the $\Delta \alpha-\Delta \delta$ plane.

The calibrated simulation code outputs show improved agreement with experimental observations, see Figure 8. The biggest improvement is visible in the moment coefficient data where the discrepancy between observations and simulations is greatly reduced by calibration. The small discrepancy that remains is caused by the fact that the simulation model is not able to exactly reproduce the observational data using the optimal calibration parameters.

The uncertainty intervals in Figure 8 indicate various types of uncertainty. The uncertainty in the uncalibrated simulations is caused by uncertainties about the true input values or variations in the inputs. The uncertainty in the calibrated simulations on the other hand is an indication for how uncertain the calibration estimates are based on the observation data and prior knowledge. Increasing the amount of data will yield a decrease in the uncertainty level that the calibration estimates are the best guess for reducing the discrepancy between simulations and observations. The measurement uncertainty is an estimate of the error that is possibly made by the measurement device.

The calibration estimates refer only to code inputs and do not represent true physical quantities, partly because the calibration parameters $\Delta \alpha$ and $\Delta \delta$ include not only uncertainties in angle of attack and flap angle but also geometric uncertainties. Furthermore, even in case the calibration parameters would reflect the experimental uncertainties as they exist in the experiment the calibration estimates do not represent physical quantities since a computer model does never exactly represent reality. 


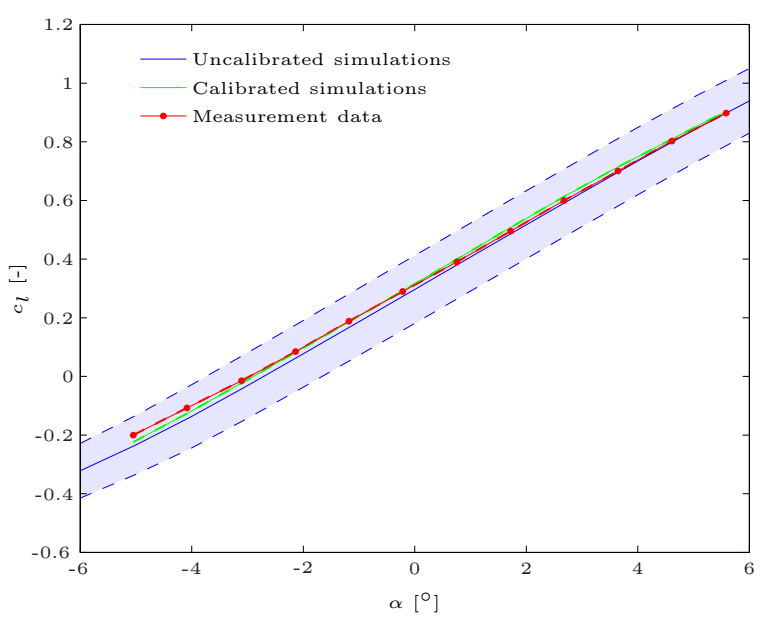

(a) Lift coefficient.

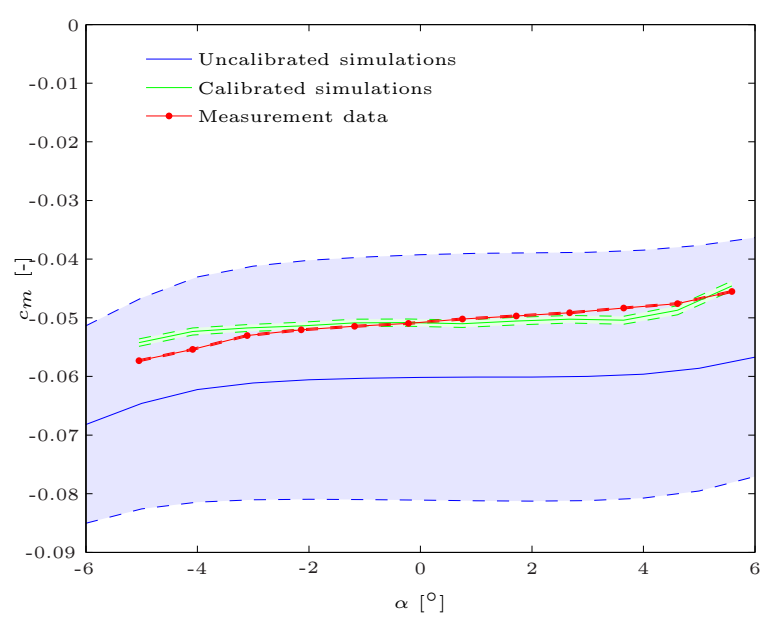

(b) Moment coefficient.

Figure 8: Comparison of the numerical simulations before and after calibration with the experimental observations. The shaded areas indicate uncertainty intervals of 1 standard deviation.

\section{IV.B. Prediction}

Predictions are made using the calibrated simulation model and compared with observations to assess the success of the calibration procedure. In experimental aerodynamics is happens regularly that some physical quantities are unmeasured. These unmeasured quantities can be predicted using a simulation model that is calibrated based on the available measured data. In this work calibration estimates are obtained from calibrating the simulation model using observational data of the lift coefficient and the moment coefficient. Another output of interest, i.e. the drag coefficient, is used to check whether the calibrated simulation model provides good agreement between numerical simulations and observations of quantities that are not used when calibrating the model. Predictions of the drag coefficient are made using the simulation model with the calibration estimates as inputs and are therefore subject only to uncertainties that remain after the calibration process in the calibration estimates. Estimates of the uncertainty in drag coefficient are obtained based on Monte Carlo simulations of the calibration estimates with a converged amount of input samples of $10^{4}$.

Figure 9 shows the prediction results of the calibrated simulation model. Although normally predictions are made because of lack of observational data, in this work observational data is available to assess the success of the calibration approach. The predicted drag shows good agreement with the experimental observations since it lies within a measurement uncertainty range of 2 standard deviations. The prediction uncertainty caused by uncertainty in the calibration estimates is negligible with respect to the measurement uncertainty. It can be concluded that the simulation model is successfully calibrated since predictions show good agreement with observations with relatively small uncertainty levels.

\section{Conclusion}

The uncertainty quantification presented in this paper provides a methodology to compare experimental observations with numerical simulations in a reliable way by taking into account the effect of input uncertainties on the outputs. The methodology is applicable to a large variety of aerodynamic problems since often when performing aerodynamic experiments the results are compared with numerical simulations. Being aware of the fact that uncertainties play often a minor role in steady aerodynamics experiments, this methodology is even more relevant when studying unsteady aerodynamics and fluid-structure interaction phenomena.

An aerodynamic experiment is performed that is subject to input uncertainties that are considered to influence the outputs significantly. Uncertainty quantification is applied to the experiment to investigate the input uncertainties and their influence on the outputs. Initially various uncertain input variables are selected that are expected to be important for the stochastic response of the outputs. Uncertainty distributions are 


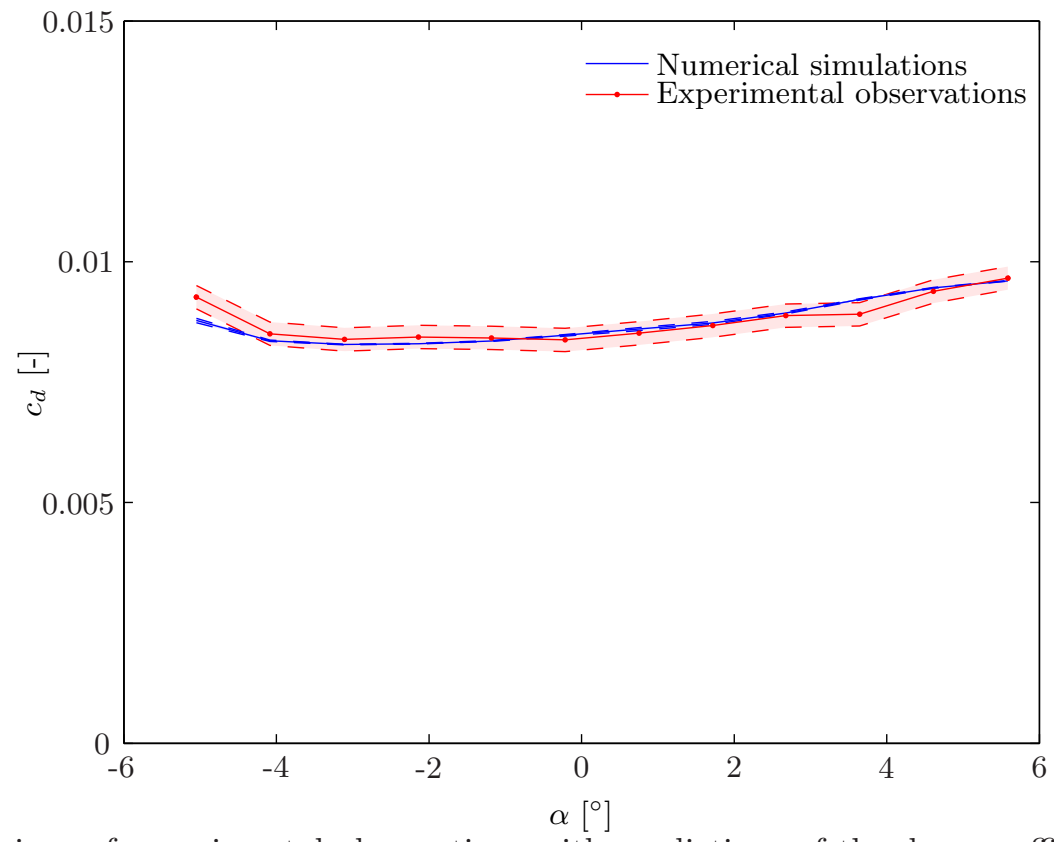

Figure 9: Comparison of experimental observations with predictions of the drag coefficient using the calibrated simulation model. The shaded areas indicate uncertainty intervals of 2 standard deviations.

derived based on measurements of the uncertain variables and measurement device tolerances. A sensitivity analysis is applied to the uncertainty distributions, which shows that the geometry, the angle of attack and the flap angle are the most important uncertain variables. The uncertainty distributions of the most important variables are propagated through the XFOIL computer simulation code to obtain the stochastic response on the outputs. Uncertainty levels expressed in standard deviations of 0.11 and 0.02 are obtained for the lift coefficient and moment coefficient respectively. The experimental observations lie within the uncertainty range of the simulations.

Bayesian calibration is employed to reduce the uncertainty levels in the numerical simulation outputs and improve on the agreement between simulations and experimental observations. The calibration is performed making use of the lift coefficient and moment coefficient data. Numerical simulations obtained from the calibrated model show considerably smaller uncertainty levels and a significant improvement in the agreement between simulations and observations. The results of the calibration are assessed by making predictions of the drag coefficient, which is not used for calibration, and comparing them with experimental observations. The predictions of the drag coefficient show good agreement with the experimental observations. Therefore, it can be concluded that the calibration procedure has proven to be successful in providing improved predictions.

In experimental aerodynamics often some physical quantities are unmeasured e.g. since they are difficult to measure. Bayesian calibration offers possibilities to predict unmeasured quantities using simulation codes by taking account of input uncertainties and the measured physical quantities. Calibrating the simulation model yields improved agreement between observations and predictions. Thus, calibration offers a more sophisticated method to conventional predictions where the desired experimental variables are used as code inputs and no account is taken of uncertainties and the experimentally observed physical quantities.

Challenges for the uncertainty quantification and data assimilation methodology are to apply it to more complex aerodynamic problems of unsteady flow and fluid-structure interaction cases. Furthermore, application of the methods to more sophisticated measurement techniques such as particle image velocimetry will be interesting, since this measurement technique provides large datasets with relatively high uncertainty levels compared to conventional force measuring techniques.

\section{References}

\footnotetext{
${ }^{1}$ Babuska, I., Nobile, F., and Tempone, R., "A Stochastic Collocation Method for Elliptic Partial Differential Equations with Random Input Data," SIAM Journal of Numerical Analysis, Vol. 45, No. 3, 2007, pp. 1005-1034.
} 
${ }^{2}$ Loeven, G. J. A., Witteveen, J. A. S., and Bijl, H., "Probabilistic Collocation: An Efficient Non-Intrusive Approach For Arbitrarily Distributed Parametric Uncertainties," Proceedings of the 45th AIAA Aerospace Sciences Meeting and Exhibit, No. AIAA paper 2007-317, Reno (NV), United States, Jan. 2007.

${ }^{3}$ Chassaing, J. C. and Lucor, D., "Stochastic Investigation of Flows About Airfoils at Transonic Speeds," AIAA Journal, Vol. 48, No. 5, May 2010, pp. 1852-1867.

${ }^{4}$ Kennedy, M. C. and O'Hagan, A., "Bayesian calibration of computer models," Journal of the Royal Statistical Society $B$, Vol. 63, 2001, pp. 425-464.

${ }^{5}$ O'Hagan, A., "Bayesian Analysis of Computer Code Outputs: A Tutorial," Reliability Engineering 85 System Safety, Vol. 91, 2006, pp. 1290-1300.

${ }^{6}$ Krytzny, A. and Ewald, B., "Conventional Wall Corrections for Closed and Open Test Sections," Wind Tunnel Wall Corrections, No. AGARD-AG-336, Oct. 1988.

${ }^{7}$ Timmer, W., "Two-dimensional low-Reynolds number wind tunnel results for airfoil NACA 0018," Wind Engineering, Vol. 32, No. 6, 2008, pp. 525-537.

${ }^{8}$ Drela, M., "XFOIL homepage," http://web.mit.edu/drela/Public/web/xfoil/, Feb. 2012.

${ }^{9}$ van Ingen, J. L., "A method of calculating the transition region for two-dimensional boundary layers with distributed suction," Tech. Rep. VTH-132, Technische Hogeschool Delft, 1965.

${ }^{10}$ Kennedy, M. C. and O'Hagan, A., "Predicting the output from a complex computer code when fast approximations are available," Biometrika, Vol. 87, 2000, pp. 1-13.

${ }^{11}$ Higdon, D., Gattiker, J., Williams, B., and Rightley, M., "Computer Model Calibration Using High Dimensional Output," Journal of the American Statistical Association, Vol. 103, 2008, pp. 570-583.

${ }^{12}$ Duvigneau, R., "Adaptive Parametrization using Free-Form Deformation for Aerodynamic Shape Optimization," Tech. rep., Unité de recherche INRIA Sophia Antipolis, France, July 2006.

${ }^{13}$ Loeven, G. J. A. and Bijl, H., "Probabilistic Collocation used in a Two-Step approach for efficient uncertainty quantification in computational fluid dynamics," Computer Modeling in Engineering E3 Science, Vol. 36, 2008, pp. 193-121. 